\title{
Exposure to robotic virtual agent affects adoption of intentional stance
}

\author{
Lorenzo Parenti \\ Italian Institute of Technology (IIT), Genoa, Italy \\ Department of Psychology, University of Turin, Turin, Italy \\ Lorenzo.parenti@iit.it \\ Serena Marchesi \\ Italian Institute of Technology (IIT), Genoa, Italy \\ School of Computer Science, Faculty of Science and Engineering, Manchester University, Manchester, United Kingdom \\ Serena.marchesi@iit.it \\ Marwen Belkaid \\ Italian Institute of Technology (IIT), Genoa, Italy \\ Marwen.belkaid@iit.it \\ Agnieszka Wykowska \\ Italian Institute of Technology (IIT), Genoa, Italy \\ Agnieszka,wykowska@iit.it
}

\begin{abstract}
Understanding how and when humans attribute intentionality to artificial agents is a key issue in contemporary human and technological sciences. This paper addresses the question of whether adopting intentional stance can be modulated by exposure to a 3D animated robot character, and whether this depends on the human-likeness of the character's behavior. We report three experiments investigating how appearance and behavioral features of a virtual character affect humans' attribution of intentionality toward artificial social agents. The results show that adoption of intentional stance can be modulated depending on participants' expectations about the agent. This study brings attention to specific features of virtual agents and insights for further work in the field of virtual interaction.
\end{abstract}

\section{CCS CONCEPTS}

- Applied computing $\sim$ Law, social and behavioral sciences $\sim$ Psychology $\bullet$ Human-centered computing $\sim$ Human computer interaction $(\mathrm{HCI}) \sim \mathrm{HCI}$ design and evaluation methods $\bullet$ Computing methodologies $\sim$ Computer graphics $\sim$ Animation

\section{Keywords:}

Virtual agent, Intentional stance, Human-likeness, Appearance

\section{INTRODUCTION}

The development of increasingly complex robotic systems, from industrial robots for manufacturing to high precision surgical robots, has a great impact on our daily lives. One area that is gaining scientific interest and popularity is social robotics, where autonomous or semi-autonomous robots have the ability to interact and communicate with humans or other autonomous agents, following social behaviors and roles [1,2]. Recent studies suggest that humans can perceive these artificial systems as social agents (for a review see [3]). However, it is still unclear what specific mechanisms of social cognition mechanisms humans employ to interpret the behavior of these new social partners [4, 5. 6].

One of the key mechanisms of human social cognition that entails perceiving other agents as social entities is the attribution of intentionality to their behaviors [7]. This process is thoroughly explained by Daniel Dennett [8, 9], who 
This is an accepted manuscript version of the paper published in: HAI '21, November 9-11, 2021, Virtual Event, Japan (C) 2021 Copyright is held by the owner/author(s). ACM ISBN 978-1-4503-8620-3/21/11.

https://doi.org/10.1145/3472307.3484667

defines the concept of "stances" (or strategies) that humans adopt to explain and predict others' behaviors. Dennett presents two stances that we can adopt: (i) the intentional stance, that is adopted when we interpret the agents' behavior with reference to mental states; and (ii) the design stance, which is adopted when we interpret the behavior of observed agents with reference to how they were designed to behave. When it comes to human agents, adopting the intentional stance is a default strategy enabling an efficient way to navigate the social environment (for a review see [10]). It still remains to be answered, however, if (and under what conditions) humans adopt the intentional stance also towards artificial agents. One of the challenges in addressing this question is how to operationalize and empirically address a philosophical concept such as intentional stance. In order to address this challenge, Marchesi and colleagues [11, 12] developed a tool (the InStance Test, IST) to assess individual tendencies to adopt the intentional or the design stance towards robots. With the use of the InStance Test, the authors found that people do sometimes adopt intentional stance towards robots, and that whether they are more likely to do so (rather than adopting the design stance) can be determined based on individual neural activity at rest [13].

However, one needs to take into consideration that robots are a special category of artificial agents, as they are physically embodied, and thus, occupy physical space in human environment. They can also modify the shared environment, by, for example manipulating objects. Therefore, the likelihood of attribution of intentionality to robots might differ from the likelihood of attribution of intentionality to other types of artificial agents. As nowadays we casually interact also with virtual agents and avatars, it remains to be answered whether intentional stance can also be adopted towards this category of artificial agents.

In this study, we aimed to examine whether human-likeness of appearance and behavior of a virtual robotic agent can affect the stance humans adopt toward robots. We created different types of $3 \mathrm{D}$ virtual characters and presented them to participants in an online experiment, probing the adoption of intentional stance before and after exposure to the artificial agents.

\section{METHODS}

\subsection{Experiments}

Three experiments were designed to measure the adoption of intentional stance and negative attitudes toward these virtual robot agents. In the following section, we report the methods and materials that are common to all three experiments and then, we report specific variants of each experiment. The experiments were implemented in SoSci Survey (www.soscisurvey.de) and were made available to users via a link embedded in Prolific.ac call to participation. The study was approved by the local Ethical Committee (Comitato Etico Regione Liguria) and was conducted in accordance with the Code of Ethics of the World Medical Association (Declaration of Helsinki).

\subsection{Participants}

Participants were recruited online through Prolific (www.prolific.ac) and paid according to Prolific.ac guidelines. All participants were at least 18, fluent in English and reported normal or corrected-to-normal vision, no history of cognitive impairment, mental illnesses or Autism Spectrum Disorder. All participants were naïve to the purpose of the study and provided informed consent prior to participation. Two-hundred individuals were recruited and the data of 195 participants were included in the final analyses (mean age $=27.7$, range $=18-64,99$ females, all right-handed). Five participants' data were not included in the analyses due to not completing the task.

\subsection{Stimuli}

The stimuli consist of animated videos of different virtual agents (VAs) implemented through Unreal Engine (Epic Games: www.unrealengine.com) VAs appearance is based on the humanoid robot iCub [14] and behaviors are implemented following Human- and Robot-like motion (see Figure 1.A). One VA is acting following the principles of biological motion and its actions are created to look as human-like as possible (for an accurate definition of biological motion see [15]). The behavior of the second VA is rather mechanistic and designed to look more machine-like (Experiment 1 and 2). As a 
This is an accepted manuscript version of the paper published in: HAI '21, November 9-11, 2021, Virtual Event, Japan (C) 2021 Copyright is held by the owner/author(s). ACM ISBN 978-1-4503-8620-3/21/11.

https://doi.org/10.1145/3472307.3484667

control, we also implemented a third VA behaving the same way as the Robot VA with human-like behavior but with the appearance of a human kid (see Figure 1.B) (Experiment 3) because the robot iCub is designed with child-like appearance. Using animated VAs allowed us to manipulate the human-likeness of the agent motion, which would have been more challenging on the real robot iCub. From the viewer perspective, VAs were both virtually located standing behind a game table, thus participants were able to see the upper body of the VA (see Figure 1.A-B). Example video clips for each VA were uploaded to a repository and available at the link: https://osf.io/rhvyu/?view_only=2a8bea70f5294581bce93dc60bd93337
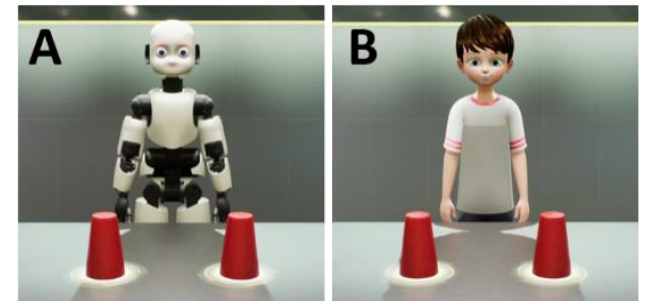

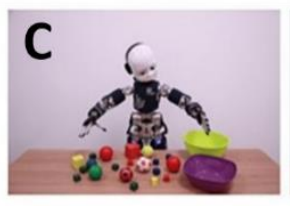

iCub knows it's better to clean the table after playing
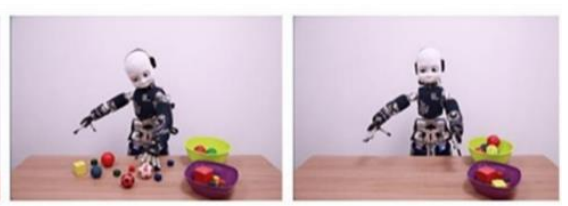

iCub places objects in bowls of the same shape

Figure 1. A: the VA with the appearance of iCub Robot. B: human-appearance version of the VA. C: Example scenario from the InStance Questionnaire, which aims to test the adoption of intentional stance towards robots.

Both the Robot VAs with human-like and mechanistic behavior performed the same type of action inside the Unreal Engine application: gazing behaviors, pointing behaviors, head-tilting behaviors, positive reactions, negative reactions and "turning-on" action resembling the robot turning-on. These behaviors were recorded in 25 different video clips for each VA. Video clips order was randomized and shown to participants one at a time. Each video clip was presented with a slider below to rate the movement in the clip. One pole of the slider is always stating "Human-like" and the opposite one "Machine-like". The rating scale was then coded so that "0" represents Machine-like (VA movement perceived as entirely machine-like), "100" represents Human-like (VA movement perceived as totally human-like) and a score of 50 represents a neutral response. Averaged ratings score for each VAs was calculated for each participant.

\subsection{Questionnaires}

Two questionnaires were administered to examine participants' subjective perceptions of the robotic agents. The first was InStance Questionnaire (IST), which measures participants' tendency to adopt Intentional Stance toward iCub [11]. The second questionnaire was the Negative Attitude Towards Robots (NARS) [16], which measures prior attitudes toward robotic agents and behavioral differences in HRI following three different sub-scales [16, 17].

To measure whether watching the robotic VA modulated participants' tendency to adopt intentional stance toward the robot, the IST was administered before and after exposure to the VA (pre- and post-). In order to avoid participants to rate twice the same items, we split the IST presentation in two halves, based on a prior work [18]. IST pre- and post- consisted of 17 items each. Each IST item consists of a scenario made of three different pictures of iCub conducting an action alone or with a human partner (see Figure 1.C). Two sentences describing the situation are presented below the scenario. One description is written with mentalistic words and the other with mechanistic terms. Participants were asked to indicate whether the mentalistic or mechanistic description was fitting the scenario better by moving a slider placed on a line between the two sentences. IST scoring was calculated by assigning 0 to the extreme of the slider line on the mechanistic side (adopting the mechanistic stance) and 100 to the extreme side of the line on the mentalistic side (adopting the intentional stance). IST pre- and post- averaged scores were calculated for each participant. IST delta scores were calculated for each participant subtracting IST pre- score from IST post- score, in order to have a unique measure for IST modulation related to exposure.

The NARS consisted of 14 items rated on a 5-point Likert scale (from 1: "I strongly disagree" to 5: "I strongly agree") and was administered at the beginning of Experiment 2. NARS was scored and averaged for each subscale individually: S1="Negative Attitude toward Situations of Interaction with Robots", S2="Negative Attitude toward Social Influence of Robots", and S3="Negative Attitude toward Emotions in Interaction with Robots" [16]. 
This is an accepted manuscript version of the paper published in: HAI '21, November 9-11, 2021, Virtual Event, Japan (C) 2021 Copyright is held by the owner/author(s). ACM ISBN 978-1-4503-8620-3/21/11.

https://doi.org/10.1145/3472307.3484667

\subsection{Procedure and Design}

\subsubsection{Experiment 1 .}

Experiment 1 was designed to assess to impact of exposure to Robot VAs with human-like and mechanistic behavior on the adoption of intentional stance. One-hundred participants were recruited and split in two groups: 50 individuals saw and rated only the Robot VA with human-like behavior and the other half, only the Robot VA with mechanistic behavior. Three participants were excluded from final analysis resulting in a final sample of 49 for the Robot VA with human-like behavior condition (mean age $=25.7$, range $=19-49,25$ females) and 48 for the Robot VA with mechanistic behavior condition (mean age $=26.2$, range $=18-39,23$ females). After reading instructions, all participants completed the IST -pre- test. Next, participants were instructed to rate each behavior of the VA (either human-like or mechanistic). After participants rated all the 25 behaviors, they completed the IST post- test.

\subsubsection{Experiment 2 .}

In order to investigate whether a between-participants design (i.e. seeing only one type of behavior) might have influenced the ratings, in Experiment 2 we designed a within-participants experiment. Fifty participants were recruited and 48 were included in the final sample (mean age $=28.5$, range $=18-58,21$ females). After reading the instructions, all participants completed the NARS then IST pre- test. Next, participants were instructed to rate behaviors as in Experiment 1. Here, all the video clips from the Robot VAs with human-like behavior and mechanistic behavior were randomly shown and rated with the same slider. After participants rated all the 50 behaviors ( 25 for each VA), they completed the IST post-test.

\subsubsection{Experiment 3 .}

As a control group, we implemented a third condition in which the 25 behaviors were performed by the Kid-appearance VA. Experimental procedure was the same as in Experiment 1. Fifty participants completed the study and none of them was excluded from the analysis (mean age $=30.08$, range $=18-64,30$ females).

\subsection{Analysis}

Data were analyzed using R version 4.0.2. (RStudio Team (2010): www.rstudio.com). The data were analyzed with regard to differences between IST pre- and post- scores: repeated measures ANOVA for Experiment 1, paired-sample t-test for Experiment 2 and 3, and ANOVA to compare the three Studies. To examine participants' differences in rating the VAs behaviors, we ran an independent t-tests for Experiment 1 where the between factor was the type of behavior participants were exposed to, a paired-sample t-test for Experiment 2, where the within factor was the type of behavior, and ANOVA to investigate differences among the three groups from Experiment 1 and 3, where the 3-levels within factor was the type of VA. Studies are designed following the idea that being exposed to different degree of human-likeness can affect attribution of intentionality. After Experiment 1, we decided to include NARS in Experiment 2 to examine how subjective negative attitudes towards robotic agents may be related to IST scores and ratings. For this reason, participants completed NARS, IST pre-, ratings and IST post- in order to later analyze how NARS scores could affect the subsequent measures, how IST pre- scores influence ratings and how exposure to the VAs affect IST post- scores. The associations between NARS and IST scores, IST scores and ratings, and NARS and ratings were investigated through separate linear regressions for each study. This analysis was not included in Experiment 3 where the stimuli did not have a robotic appearance. Throughout the paper, multiple comparisons were corrected and p-values were reported according to Tukey's correction. Cohen's d and eta-squared equations were used to calculate effect sizes respectively for t-test and ANOVA.

\section{RESULTS}

\subsection{Experiment 1}

First, we examined if the two groups were different based on demographical information. The t-test showed no differences between the two groups based on age $(\mathrm{t}(95)=-0.385, \mathrm{p}=0.701$, Cohen's $\mathrm{d}=-0.078)$. The Chi-Squared analysis showed no differences based on sex $\left(\mathrm{X}^{2}=0.093, \mathrm{p}=0.760\right)$ or student status $\left(\mathrm{X}^{2}=0.009, \mathrm{p}=0.923\right)$. 
This is an accepted manuscript version of the paper published in: HAI '21, November 9-11, 2021, Virtual Event, Japan (C) 2021 Copyright is held by the owner/author(s). ACM ISBN 978-1-4503-8620-3/21/11.

https://doi.org/10.1145/3472307.3484667

\subsubsection{IST scores.}

Secondly, we submitted IST individually averaged scores to a repeated measures two-way ANOVA with groups as a between-subject factor and IST block as a within-subject factor. The results showed a within-subjects effect of IST score $\left(\mathrm{F}(1,95)=4.109, \mathrm{p}=0.045, \eta^{2}=0.004\right)$ and no effect of the group $(\mathrm{p}=0.877)$ or interaction $(\mathrm{p}=0.377)$. This difference $\left(\mathrm{M}_{\text {IST-pre }}=35.416 \mathrm{vs}\right.$ MIST-post $\left.=38.025\right)$ was investigated with post-hoc t-test showing a significant difference of IST pre and post score in the Robot VA with mechanistic behavior group $(t(47)=-2.116, p=0.04$, Cohen's $d=-0.305)$ but not in the Robot VA with human-like behavior group $(\mathrm{t}(48)=-0.787, \mathrm{p}=0.435$, Cohen's $\mathrm{d}=-0.153$ ) (see Figure 2).

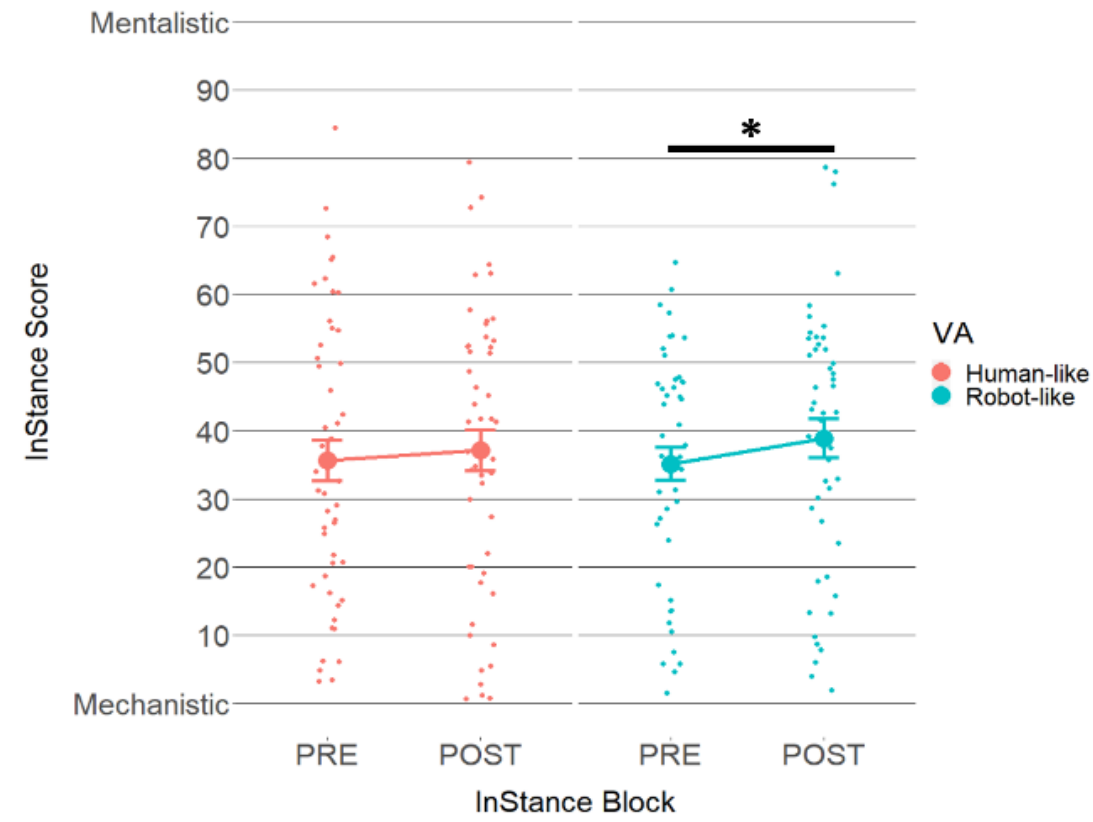

Figure 2. Differences in InStance Questionnaire scores between the two groups of Experiment 1.

\subsubsection{Human-likeness ratings.}

We performed a t-test to compare averaged ratings showing that Robot VA with human-like behavior is rated as more human-like $(\mathrm{M}=55.122)$ and Robot VA with mechanistic behavior as more machine-like $(\mathrm{M}=45.836)(\mathrm{t}(95)=2.948, \mathrm{p}$ $=0.004$, Cohen's $d=0.599)$. The exploratory correlation between IST scores and ratings showed no significant correlations (ratings - IST Pre: $r(97)=0.151 \mathrm{p}=0.141 ;-$ IST Post: $\mathrm{r}(97)=0.162, \mathrm{p}=0.113$ ).

\subsection{Experiment 2}

\subsubsection{IST scores.}

The t-test examining differences between IST pre- and post- scores showed a significant effect $(\mathrm{t}(47)=-3.234, \mathrm{p}=0.002$, Cohen's $d=-0.467)$, where the mean of the IST pre- score $(M=28.201)$ was lower than IST post- score $(M=34.540)$.

\subsubsection{Human-likeness ratings.}

Ratings related to the Robot VA with human-like behavior and Robot VA with mechanistic behavior were compared and submitted to a paired-sample t-test $(\mathrm{t}(47)=8.860, \mathrm{p}<0.001$, Cohen's $\mathrm{d}=1.279)$, showing that Robot VA with mechanistic behavior was rated as more machine-like $(\mathrm{M}=36.291)$ compared to the Robot VA with human-like behavior $(\mathrm{M}=54.438)$. A linear regression was performed to predict ratings and IST scores based on NARS sub-scales score. NARS sub-scale 3 predicted IST pre- scores $(\mathrm{F}(1,46)=5.02, \mathrm{p}=0.03)$ with an R2 of 0.098 and IST post- $(\mathrm{F}(1,46)=5.686, \mathrm{p}=0.021)$ with 
This is an accepted manuscript version of the paper published in: HAI '21, November 9-11, 2021, Virtual Event, Japan (C) 2021 Copyright is held by the owner/author(s). ACM ISBN 978-1-4503-8620-3/21/11.

https://doi.org/10.1145/3472307.3484667

an R2 of 0.11. Moreover, NARS sub-scale 2 predicted the ratings for the Robot-like condition $(F(1,46)=5.331, p=0.025)$ with an R2 of 0.104 . No other significant associations were found among IST, NARS and ratings.

\subsection{Experiment 3}

The paired sample t-test examining differences between IST pre- and post- scores showed a non-significant effect $(\mathrm{t}(49)=$ $-1.149, \mathrm{p}=0.256$, Cohen's $\mathrm{d}=-0.162$ ). No significant associations were found between IST and ratings.

\subsection{Comparisons across experiments}

We first examined if the four groups taken together (2 from Experiment 1, 1 from Experiment 2 and 1 from Experiment 3) showed differences in demographic variables and scored differently in IST and ratings. The ANOVA showed no differences related to age $\left(\mathrm{F}(3,191)=2.469, \mathrm{p}=0.063, \eta^{2}=0.037\right)$ and Chi-Squared test revealed no difference regarding sex $\left(\mathrm{X}^{2}=2.808, \mathrm{p}=0.422\right)$. Results of the ANOVA examining differences in IST scores showed no effect for IST pre- scores $\left(F(3,191)=1.762, p=0.156, \eta^{2}=0.027\right)$ or IST post- scores $\left(F(3,191)=0.594, p=0.620, \eta^{2}=0.009\right)$. Next, we submitted IST delta scores to an ANOVA showing no significant differences among the groups $(\mathrm{F}(3,191)=$ $1.306, \mathrm{p}=0.274, \eta^{2}=0.02$ ).

Finally, we run t-tests to compare ratings of VAs behaviors between Experiment 1 and Experiment 2, showing a significant difference for Robot VA with mechanistic behavior ratings $(\mathrm{t}(94)=-3.168, \mathrm{p}=0.002$, Cohen's $\mathrm{d}=-0.647)$ where Experiment 1 mechanistic behavior was rated as less machine-like $(M=45.836)$ compared to Experiment $2(M=36.291)$. No difference in ratings was found in the Robot VA with human-like behavior group $(\mathrm{t}(95)=-0.236, \mathrm{p}=0.814$, Cohen's $\mathrm{d}=-0.048)$. We run a one-way ANOVA to examine differences in ratings associated with the type of VA between the two between-subject studies (Experiment 1 and Experiment 3). The analysis showed a significant effect of the VA type on ratings $\left(\mathrm{F}(2,144)=13.883, \mathrm{p}<0.001, \eta^{2}=0.162\right)$ as shown in Figure 3.

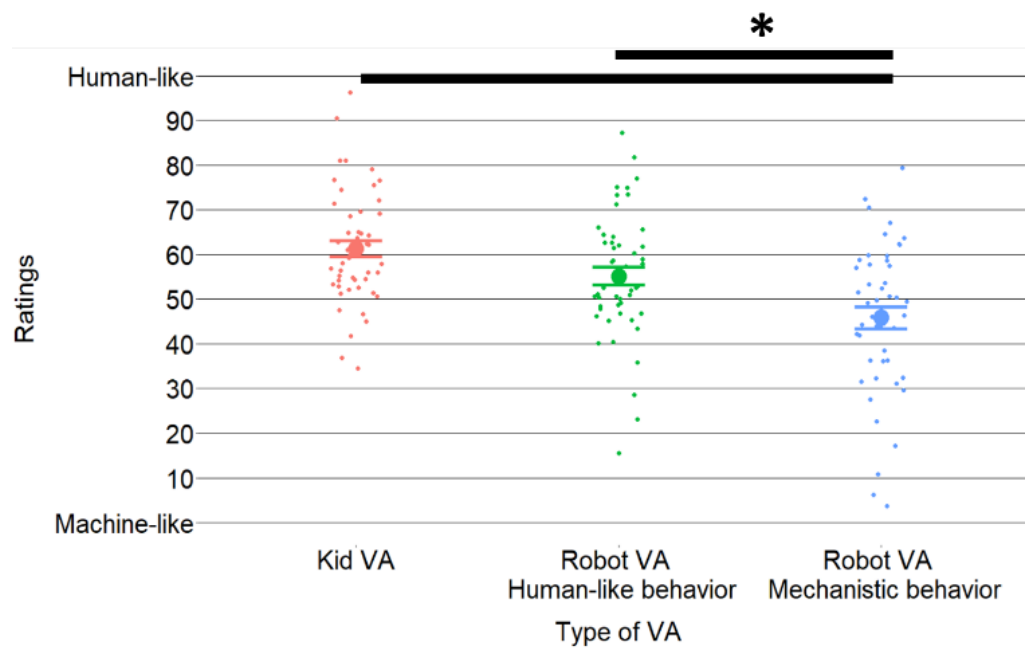

Figure 3. Differences in ratings of Human-likeness among the three groups of Experiment 1 and 3.

Post hoc comparisons highlighted significant differences in ratings between Kid and Robot VA with mechanistic behavior $(\mathrm{t}=3.133, \mathrm{p}=0.006)$ and between the two robots (with Human- and mechanistic behavior respectively) $(\mathrm{t}=5.241, \mathrm{p}<$ 0.001). No significant differences were found between Kid VA and robot with human-like behavior ratings $(p=0.093)$. 
This is an accepted manuscript version of the paper published in: HAI '21, November 9-11, 2021, Virtual Event, Japan (C) 2021 Copyright is held by the owner/author(s). ACM ISBN 978-1-4503-8620-3/21/11.

https://doi.org/10.1145/3472307.3484667

\section{General Discussion}

The main goal of this study was to assess whether the adoption of intentional stance could be modulated by exposure to a 3D animated robot avatar, and whether this depends on the human-likeness of the character's behavior. To do so, we asked participants to watch different video clips displaying animations of a virtual robot and tested whether this affected their tendency to interpret robots' behavior in mentalistic terms using IST [11, 12]. The human-likeness of the virtual robot behavior was manipulated by designing the animations such that they would show either human-like or machine-like motions.

Overall, participants showed a bias toward a mechanistic explanation when they were asked to judge robot actions in the IST (mean from the 3 experiments of IST pre score $=36.721$ ). This is consistent with previous studies [11, 19]. Interestingly however, our results show that IST scores increase after exposure to the robot with mechanistic behaviors but not to the robot with human-like behaviors. In other words, machine-like behaviors of the robotic virtual agent had an effect on participants' adoption of intentional stance. While this result may seem counter-intuitive, it is in line with previous studies where the IST scores increased when participants' expectations about the robot were met [20]. In our study, it is likely that participants expected that a virtual agent with the appearance of a robot act in a robotic way, even if it is digitally embodied. Only when this expectation was met, were they more inclined to adopt a more intentional stance. On the other hand, exposure to the same human-like movements implemented on the Kid-appearance VA (Experiment 3) did not affect IST score.

In addition to filling out the IST, participants were asked to rate each video clip with respect to machine-likeness vs. human-likeness. As expected, participants rated the behavior of the robot with mechanistic behavior as more machine-like, and vice versa for the robot with human-like behavior. Exposing participants to both VAs (Experiment 2) resulted in even more polarized ratings where the robot with mechanistic behavior rated as more machine-like compared to the same condition in Experiment 1. In addition, there was no significant difference between the rating of the robot with human-like behavior and the Kid VA - even though the human-likeness ratings for the Robot VA with human-like behavior were slightly lower than for the Kid VA. This demonstrates that human-likeness of behavior can be manipulated independently from appearance: the same type of behavior generated similar ratings when implemented on a human and robot character while different types of behavior were rated differently even though they were exhibited by the same agent. Taken together with IST scores, these results further highlight that, while seemingly close concepts, intentionality and human-likeness are two distinct dimensions when it comes to how humans perceive the behavior and appearance of artificial social agents.

We included NARS in Experiment 2 to have a measure of subjective attitude toward robots and robotic agents. Specifically, NARS sub-scale 2 includes items related to the social influence of robots and it was named "negative attitudes toward social influence of robots". NARS sub-scale 3 includes items associated to emotions evoked in interaction with the robots and thus was named "negative attitudes toward emotions in interaction with robots" [17]. Interestingly, higher scores in NARS sub-scale 2 predicted more machine-like ratings for the robot VA with mechanistic behavior. This could be due to believing that robots could be associated with negative cultural and societal outcomes. This could have led participants to perceive them as more machine-like and possibly more in line with their expectations or concerns. On the other hand, higher NARS sub-scales 3 scores predicted lower IST scores, for both pre- and post- tests. Thus, it seems that negative attitudes toward potential interaction with robots or robotic agents could lead participants to use more mechanistic descriptions to explain robot behaviors.

In conclusion, this work provides insights into the conditions under which humans adopt intentional stance towards virtual agents with different appearances and behaviors. Prior studies showed how factors such as appearance, repetitive behavior and exposure to robots can influence attribution of intentionality [19, 21]. In this paper, we showed how adoption of intentional stance can be influenced by the human-likeness of the agent's behavior and appearance, showing a dissociation between these two factors, and a dissociation between the attribution of intentionality and attribution of human-likeness.

\section{ACKNOWLEDGMENT}

This work has received support from the European Research Council under the European Union's Horizon 2020 research and innovation program, ERC Starting grant, G.A. number: ERC2016-StG-715058, awarded to Agnieszka Wykowska. 
This is an accepted manuscript version of the paper published in: HAI '21, November 9-11, 2021, Virtual Event, Japan (C) 2021 Copyright is held by the owner/author(s). ACM ISBN 978-1-4503-8620-3/21/11.

https://doi.org/10.1145/3472307.3484667

The content of this paper is the sole responsibility of the authors. The European Commission or its services cannot be held responsible for any use that may be made of the information it contains.

\section{REFERENCES}

[1] Sheridan, T. B. (2020). A review of recent research in social robotics. Current opinion in psychology, 36, 7-12.

[2] Bartneck, C., \& Forlizzi, J. 2004. A design-centred framework for social human-robot interaction. In RO-MAN 2004. 13th IEEE international workshop on robot and human interactive communication (IEEE Catalog No. 04TH8759) (pp. 591-594). IEEE.

[3] Hortensius, R., \& Cross, E. S. 2018. From automata to animate beings: the scope and limits of attributing socialness to artificial agents. Annals of the New York Academy of Sciences, 1426(1), 93-110.

[4] Ghiglino, D. \& Wykowska, A. 2020. When Robots (pretend to) Think. In Artificial Intelligence: Reflections in Philosophy, Theology, and the Social Sciences. Goecke P.B., and Rosenthal-von der Pütten, A. M. (Eds). Mentis.

[5] Kompatsiari, K., Ciardo, F., Tikhanoff, V., Metta, G., \& Wykowska, A. 2019. It's in the eyes: The engaging role of eye contact in HRI. International Journal of Social Robotics, 1-11.

[6] Thellman, S., Silvervarg, A., Gulz, A., \& Ziemke, T. 2016, September. Physical vs. virtual agent embodiment and effects on social interaction. In International conference on intelligent virtual agents (pp. 412-415). Springer, Cham.

[7] Frith, C. D., \& Frith, U. 2012. Mechanisms of social cognition. Annual review of psychology, 63, 287-313.

[8] Dennett, D. C. 1971. Intentional systems. The Journal of Philosophy, 68(4), 87-106.

[9] Dennett, D. C. 1989. The intentional stance. MIT press.

[10] Perez-Osorio, J., \& Wykowska, A. 2019. Adopting the intentional stance towards humanoid robots. Springer Tracts in Advanced Robotics. https://doi.org/10.1007/978-3-030-17974-8_10

[11] Marchesi, S., Ghiglino, D., Ciardo, F., Perez-Osorio, J., Baykara, E., \& Wykowska, A. 2019. Do we adopt the intentional stance toward humanoid robots?. Frontiers in psychology, 10, 450.

[12] Marchesi, S., Spatola, N., Perez-Osorio, J., \& Wykowska, A. 2021, March. Human vs Humanoid. A behavioral investigation of the individual tendency to adopt the intentional stance. In Proceedings of the 2021 ACM/IEEE International Conference on Human-Robot Interaction (pp. 332-340).

[13] Bossi, F., Willemse, C., Cavazza, J., Marchesi, S., Murino, V., \& Wykowska, A. 2020. The human brain reveals resting state activity patterns that are predictive of biases in attitudes toward robots. Science robotics, 5(46).

[14] Metta, G., Sandini, G., Vernon, D., Natale, L., \& Nori, F. 2008, August. The iCub humanoid robot: an open platform for research in embodied cognition. In Proceedings of the 8th workshop on performance metrics for intelligent systems (pp. 50-56).

[15] Thompson, J., \& Parasuraman, R. 2012. Attention, biological motion, and action recognition. Neuroimage, 59(1), 4-13.

[16] Nomura, T., Kanda, T., Suzuki, T., \& Kato, K. 2004, September. Psychology in human-robot communication: An attempt through investigation of negative attitudes and anxiety toward robots. In RO-MAN 2004. 13th IEEE International Workshop on Robot and Human Interactive Communication (IEEE Catalog No. 04TH8759) (pp. 35-40). IEEE.

[17] Nomura, T., Kanda, T., \& Suzuki, T. 2006. Experimental investigation into influence of negative attitudes toward robots on human-robot interaction. Ai \& Society, 20(2), 138-150.

[18] Spatola, N., Marchesi, S., \& Wykowska, A. 2021, May 28. The Instance Task: how to measure the mentalistic bias in human-robot interaction. https://doi.org/10.31234/osf.io/b3wtq

[19] Wiese, E., Metta, G., \& Wykowska, A. 2017. Robots as intentional agents: using neuroscientific methods to make robots appear more social. Frontiers in psychology, 8,1663 .

[20] Perez-Osorio, J., Marchesi, S., Ghiglino, D., Ince, M., \& Wykowska, A. 2019. Correction to: More Than You Expect: Priors Influence on the Adoption of Intentional Stance Toward Humanoid Robots. In International Conference on Social Robotics (pp. C1-C1). Springer, Cham.

[21] Abubshait, A., \& Wykowska, A. 2020. Repetitive Robot Behavior Impacts Perception of Intentionality and Gaze-Related Attentional Orienting. Frontiers in Robotics and AI, 7, 150. 